\title{
La evaluación y el turismo sostenible, caso de estudio
}

\section{Llangahua}

\section{Evaluation and sustainable tourism, Llangahua case study}

1 Yadira Alexandra Proaño Gómez https://orcid.org/0000-0003-1189-5316 Universidad Técnica de Ambato, Carrera de Turismo, Tungurahua ya.proano@uta.edu.ec

2 María Fernanda Viteri Toro https://orcid.org/0000-0003-2600-7115 Universidad Técnica de Ambato, Carrera de Turismo, Tungurahua mf.viteri@uta.edu.ec

3 Camilo Francisco Torres Oñate (iD) https://orcid.org/0000-0002-9411-5401 Universidad Técnica de Ambato, Carrera de Turismo, Tungurahua cf.torres@uta.edu.ec

4 Xiomara Abigail Chisag Andachi https://orcid.org/0000-0002-1924-4844 Universidad Técnica de Ambato, Carrera de Turismo, Tungurahua xiomarachisag@gmail.com (2023). La evaluación y el turismo sostenible, caso de estudio Llangahua. ConcienciaDigital, $6(1.4)$, https://doi.org/10.33262/concienciadigital.v6i1.4.1997

CONCIENCIA DIGITAL, es una Revista Multidisciplinar, Trimestral, que se publicará en soporte electrónico tiene como misión contribuir a la formación de profesionales competentes con visión humanística y crítica que sean capaces de exponer sus resultados investigativos y científicos en la misma medida que se promueva mediante su intervención cambios positivos en la sociedad. https://concienciadigital.org .

La revista es editada por la Editorial Ciencia Digital (Editorial de prestigio registrada en la Cámara Ecuatoriana de Libro con No de Afiliación 663) www.celibro.org.ec 


\section{Palabras}

claves:

evaluación, modelo de gestión, sostenible, comunitario.

Keywords: evaluation, management model, sustainable, community.

\section{Resumen}

Introducción. La presente investigación está enfocada en el centro de turismo Llangahua, el que se considera desde un manejo comunitario por desarrollar sus actividades en el área de páramo que colectivamente se conserva y que desde inicios del 2021 cuenta con afluencia de visitantes. Objetivo. Estructurar un modelo de gestión sostenible en base a la evaluación del centro de turismo Llangahua. Metodología. Es inductiva, de tipo básico, bajo un enfoque cualitativo, comprende un diseño descriptivo de investigación acción práctico, sustentado en un estudio bibliográfico - documental que es base para un estudio longitudinal, desarrollado con el planteamiento de tres etapas: un diagnóstico situacional; propuesta de un modelo de gestión; $\mathrm{y}$, evaluación posterior a la intervención, estableciéndose un análisis entre el antes (evaluación 1) y después de la intervención (evaluación 2), utilizando tablas cruzadas y la triangulación hermenéutica. Resultados. El trabajo permite evidenciar que la evaluación de la administración da origen al modelo de gestión sostenible del centro de turismo Llangahua, donde se ha planteado la filosofía, orgánico estructural, estrategias, programas y acciones tácticas que incluyen sesenta y cuatro instrumentos. Conclusión. La evaluación fortalece la revisión de los logros de la administración del centro de turismo y permite la toma de decisiones para el mejoramiento y sostenibilidad del centro de turismo Llangahua.

Abstract

Introduction. This research is focused on the Llangahua tourism center, which is considered from a community management to develop its activities in the paramo area that is collectively conserved and that since the beginning of 2021 has an influx of visitors. Objective. Structure a sustainable management model based on the evaluation of the Llangahua tourism center. Methodology. It is inductive, of a basic type, under a qualitative approach, it comprises a descriptive design of practical action research, supported by a bibliographic-documentary study that is the basis for a longitudinal study, developed with a three-stage approach: a situational diagnosis; proposal of a management model; and, evaluation after the intervention, establishing an analysis between before (evaluation 1) and after the intervention (evaluation 
2), using cross tables and hermeneutical triangulation. Results. The work shows that the evaluation of the administration gives rise to the sustainable management model of the Llangahua tourism center, where the philosophy, structural organic, strategies, programs and tactical actions that include sixty-four instruments have been raised. Conclusion. The evaluation strengthens the review of the achievements of the tourism center administration and allows decision-making for the improvement and sustainability of the Llangahua tourism center.

\section{Introducción}

El presente trabajo sustenta su relevancia en la evaluación para la estructuración de un modelo de gestión con instrumentos para la administración: operación, seguimiento y control, del turismo comunitario sostenible, considera según Pearce (2016a, pp. 1-16), la necesidad de gestionar el turismo para hacerlo sustentable y competitivo.

Llangahua está ubicada a $42 \mathrm{Km}$ de la ciudad de Ambato, en la parroquia Pilahuín (Solís, 2017), su hidrografía aporta con su caudal a la represa (Luna \& Polo, 2009), está a una altitud superior a los 3800 m.s.n.m. La comunidad es parte del pueblo Tomabela (Guamán \& Fabara, 2019), cuenta con 415 jefes de hogar y más de 1500 habitantes. Forma parte del Fondo de Páramos Tungurahua y Lucha contra la Pobreza a través de la Corporación de Organizaciones Populares y Campesinas Cristóbal Pajuña - COCP (Unidad de Movimientos Indígenas y Campesinos de Tungurahua, 2016).

Las lagunas de Chuquibanza y Molinococha; y, el bosque nativo de polylepis constituyen elementos naturales de este páramo que han sido complementados con servicios de alojamiento en cabañas y carpas, alimentación, cabalgatas, senderismo, pesca deportiva, cicloturismo y un salón auditorio, además en la zona se encuentra una panadería comunitaria, centro de acopio de leche y producción de derivados lácteos, infraestructura lograda gracias al fondo asignado cuando ganaron el Premio Verde en el 2014 en base al proyecto de manejo de las 6312 hectáreas que integran los páramos protegidos por la comunidad (Moreta, 2019). Este equipamiento tiene varios años y ha pasado de estar bajo la administración de una organización no gubernamental, como parte de los planes de manejo de páramos, a una administración por parte de la comunidad.

El centro turístico ha funcionado parcialmente, pero a inicios del 2021 ha tenido afluencia de excursionistas y turistas, porque existen nuevas necesidades de los visitantes potenciales y efectivos que buscan espacios en medio de la naturaleza para la recreación en base a la percepción de bioseguridad porque la pandemia COVID-19 ha generado desestabilización social, económica y psicoemocional (UNWTO, 2020), mermando en los resultados de la actividad turística a nivel de tazas de alojamiento en la zona urbana, 
contrario a esto, el excursionismo ha crecido por un cambio en la necesidad de los ecuatorianos, quienes debido a la pandemia han modificado sus formas de consumo y buscan el disfrute en espacios naturales abiertos, lo que se ha evidenciado en Llangahua, sobrepasando las expectativas, por lo que es imperante que la toma de decisiones fortalezcan el turismo rural pensado a largo plazo, considerando que es una zona de conservación natural comunitaria y que el centro requiere de una administración estable.

En los últimos años el turismo comunitario ha incrementado su relevancia en los países en vías de desarrollo porque contribuye a generar nuevas oportunidades de trabajo y progreso en las zonas rurales (Del Barco, 2010). En el 2020 el H. Gobierno Provincial de Tungurahua contrató la consultoría para la estructuración de nuevos productos turísticos, identificándose en el informe, necesidades como la búsqueda de alternativas para la administración, operación, comercialización, fortalecimiento organizativo y guiones, para garantizar la calidad del servicio en la comunidad Llangahua (Castellanos, 2021).

La Oficina Internacional del Trabajo (OIT) en su publicación de Serie Red de Turismo Sostenible Comunitario para América Latina (REDTURS), expresa que el turismo comunitario es una forma de organización empresarial sustentada en la propiedad y la autogestión de los recursos patrimoniales comunitarios, con prácticas democráticas y solidarias en el trabajo y en la distribución de los beneficios generados por la prestación de servicios turísticos, con miras a fomentar encuentros interculturales de calidad con los visitantes (Ruiz \& Masache, 2018). La Declaración de Otavalo (septiembre 2001) destacó la necesidad de promover las expresiones de la cultura de los pueblos indígenas como una fuente de diferenciación y competitividad. La Declaración de San José (octubre 2003) se pronunció por la autogestión del turismo y el protagonismo de las comunidades en la planificación, desarrollo y supervisión de las actividades en sus territorios (Oficina Internacional de Trabajo, 2006).

El turismo comunitario es una forma de gestión que impulsa la inclusión social, promueve el mejoramiento de capacidades de liderazgo, conocimientos organizacionales y competencias turísticas para el "buen vivir" de los involucrados, animando la administración responsable de los recursos naturales, humanos y culturales y la revalorización del patrimonio (Huertas, 2016).

Las buenas prácticas evidencian la relación de la política de un emprendimiento con el desarrollo sostenible por lo que está a nivel de decisiones y de acciones de una organización, evidenciando su empatía con el medio ambiente y con la sociedad, pero también son económicamente viables porque previenen, corrigen y mejoran el quehacer en este caso turístico (Municipio de Copacabana, 2008). Según el 41 Congreso de la International Association of Scientific Experts in Tourism (citado por Cardoso, 2006, pp. 5-21), el turismo sostenible es "un turismo que mantiene un equilibrio entre los intereses sociales, económicos y ecológicos, integrando las actividades económicas y recreativas 
con el objeto de buscar la conservación de los valores naturales y culturales."

Las áreas principales de la agenda 2030 son personas, prosperidad, planeta, paz y alianzas desde estas áreas se derivan los 17 objetivos de desarrollo sostenible con sus indicadores UNESCO ( 2021), pero a pesar de que existe la normativa (Beltrán et al., 2018, p.42), establecen que no se aplica por parte de los prestadores de servicios turísticos siendo importante lo expuesto por Sarasa (2014, p.104), quien considera la evaluación como punto de partida para el planteamiento de una propuesta de un proyecto de turismo rural que pueda conducir al desarrollo integrado del territorio.

Evaluar los valores asociados a la cultura organizacional permite identificar la gestión y desarrollo apoyando al mejoramiento del control para la toma de decisiones y logro de los objetivos y metas de la organización turística (Cordero et al., 2012, pp.73-74). La evaluación implica generar información acerca de un desempeño, requiere de criterios para medir los resultado (Falcão, 2015), es un proceso valioso para mejorar la eficiencia, la calidad de gestión y la efectividad (Costa \& Castanhar, 2003), es así que la evaluación de la competitividad (EC) facilita vigilar, calificar y tomar decisiones de mejoramiento para el logro de ventajas competitivas (Alonso, 2010, pp.201-202); y, evaluar la gestión turística permite proponer un modelo de gestión para fortalecer la oferta (Cayatopa, 2018, pp.77-87).

\section{Metodología}

La propuesta metodológica inductiva es de tipo básico, bajo un enfoque cualitativo, comprende un diseño descriptivo de investigación acción práctico, sustentado en un estudio bibliográfico - documental que es base para un estudio longitudinal, desarrollado con el planteamiento de tres etapas según García (2017), un diagnóstico situacional que incluye la evaluación de la administración del centro turístico y análisis de la gestión; la formalización de un modelo de gestión a través de una adaptación a los propuesto por Pearce (2016b) y una evaluación posterior a la intervención, estableciéndose un análisis entre el antes (evaluación 1) y después de la intervención (evaluación 2), utilizando tablas cruzadas y la triangulación hermenéutica en base a una investigación bibliográfica, un proceso de síntesis de la información y de análisis interpretativo; que implica explorar las fuentes, realizar una lectura discriminatoria y analizar para establecer conclusiones.

La etapa 1 corresponde al diagnóstico o etapa de evaluación 1. La información generada se recoge en la investigación de campo, habiéndose seleccionado un instrumento estandarizado del Consejo Mundial de Turismo Sostenible que gestiona los criterios como estándares mundiales para la sostenibilidad en viajes y turismo, los criterios son el mínimo, no el máximo que se deben alcanzar para abordar la sostenibilidad social, ambiental, cultural y económica (Global Sustainable Tourism Council, 2019). Los criterios son cuatro: 


\section{Figura 1}

Criterios como estándares mundiales para la sostenibilidad en viajes y turismo
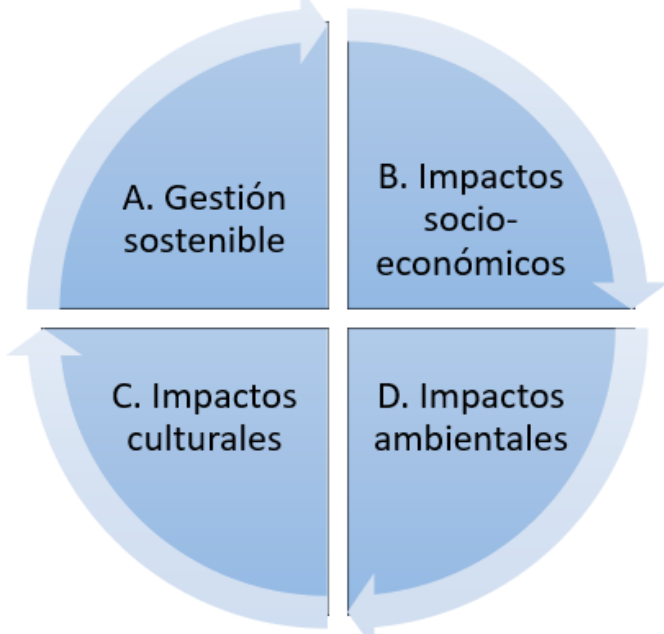

Nota: La figura representa los cuatro criterios del turismo sostenible, cada uno contiene subcriterios e indicadores.

Fuente: Global Sustainable Tourism Council (2019)

Cada criterio incluye varios subcriterios e indicadores que permiten identificar evidencias de las acciones administrativas, así como necesidades.

A. Gestión sostenible: Evalúa la estructura y marco de gestión; participación de los interesados; y, cómo se maneja la presión y el cambio:

\section{Figura 2}

\section{Criterio gestión sostenible}

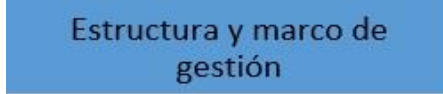

- Responsabilidad de la gestión del destino

- Estrategia de gestión de destinos y plan de acción

-Seguimiento y

presentación de informes

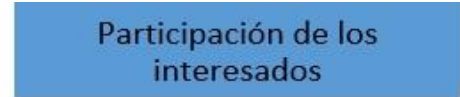

- Compromiso empresarial y normas de sostenibilidad

- Participación y comentario sde los residentes

-Participación y comentarios de los visitantes

-Promoción e informacion

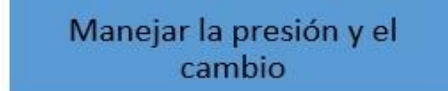

- Gestionar volumen y actividades de visitantes - Normativa urbanística y desarrollo control

-Adaptación al cambio climático

-Gestión de riesgos y crisis

Nota: El criterio gestión sostenible tiene tres subcriterios con indicadores que, en el instrumento se verifican a través del cumplimiento de cincuenta descriptores.

Fuente: Global Sustainable Tourism Council (2019)

B. Sostenibilidad socioeconómica: Evalúa la entrega de beneficios económicos locales; y, bienestar e impactos sociales. 


\section{Figura 3}

Criterio sostenibilidad socioeconómica

Entrega de beneficios
económicos locales
- Midiendo la contribución
económica de turismo
- Trabajo decente y
oportunidades profesionales
- Apoyando a los
emprendedores locales y
justocomercio

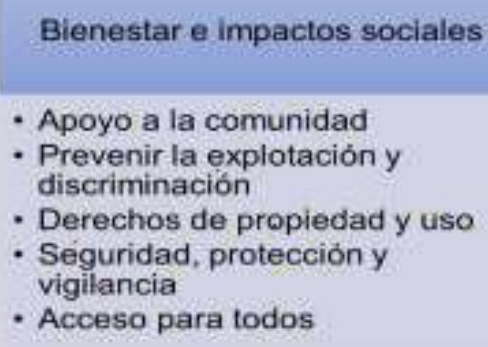

Nota: El criterio sostenibilidad socioeconómica tiene dos subcriterios con indicadores que, en el instrumento se verifican a través del cumplimiento de treinta y dos descriptores. Fuente: Global Sustainable Tourism Council (2019)

C. Sostenibilidad cultural: Evalúa la protección del patrimonio cultural; y, visitar sitios culturales.

\section{Figura 4}

\section{Criterio sostenibilidad cultural}

\section{Protección del patrimonio cultural}

-Protección de bienes culturales

-Artefactos culturales

-Patrimonio inmaterial

- Acceso local

-Propiedad intelectual

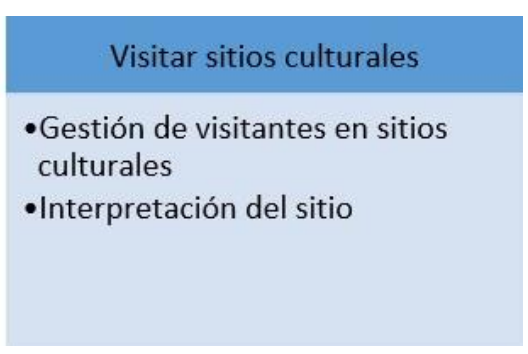

Nota: El criterio sostenibilidad cultural tiene dos subcriterios con indicadores que, en el instrumento se verifican a través del cumplimiento de veintiséis descriptores. Fuente: Global Sustainable Tourism Council (2019)

D. Sostenibilidad del medio ambiente: Evalúa la conservación del patrimonio natural; administración de recursos; y, gestión de residuos y emisiones.

\section{Figura 5}

\section{Criterio sostenibilidad del medio ambiente}
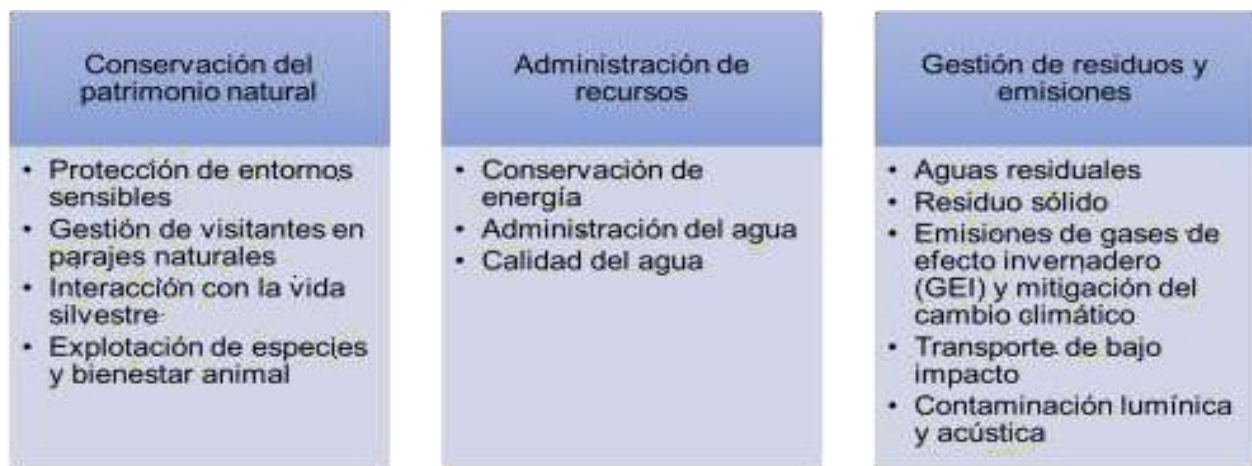

Nota: El criterio sostenibilidad del medio ambiente tiene tres subcriterios con indicadores que, en el instrumento se verifican a través del cumplimiento de sesenta y cinco descriptores.

Fuente: Global Sustainable Tourism Council (2019) 
La etapa 2 desarrolla el modelo de gestión mediante una adaptación de la metodología Modelo de gestión de destinos, que se basa en cuatro dimensiones: enfoque, objetivos, funciones y estructura organizativa Pearce (2016a), por lo que los pasos para establecer el modelo son:

\section{Figura 6}

Adaptación del modelo de gestión de destinos de Pearce

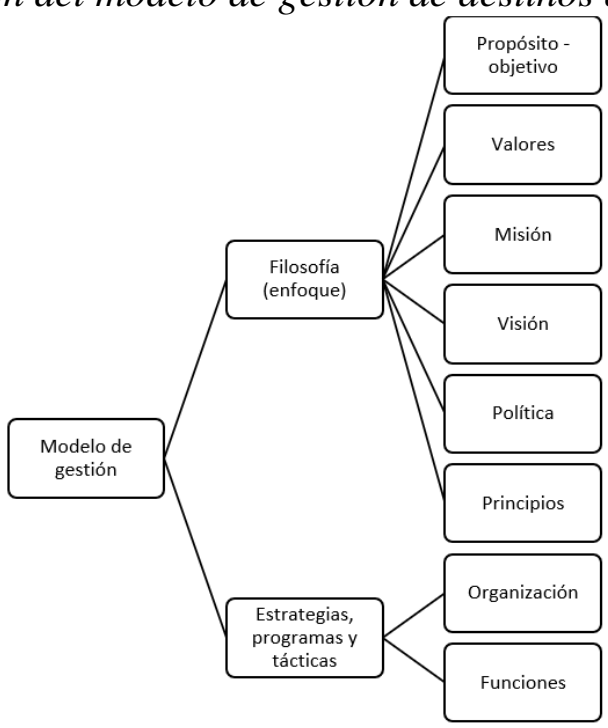

Nota: El esquema representa una modificación al modelo de gestión de destinos de Pearce, a través de dos dimensiones, sin omitir elementos del esquema original.

Fuente: Pearce (2016b)

La etapa 3 corresponde a la evaluación post intervención o etapa de evaluación 2 donde en la investigación de campo se aplica el instrumento estandarizado seleccionado tanto para el diagnóstico como para la etapa de evaluación posterior a la intervención, datos que permiten el análisis entre el antes y después de la intervención

\section{Resultados}

En la investigación realizada para estructurar un modelo de gestión sostenible en base a la evaluación del centro de turismo Llangahua, se obtuvieron los siguientes resultados:

Tabla 1

Tabla cruzada de verificación del criterio gestión sostenible

\begin{tabular}{lllr|r|r}
\hline & & No & Si & \multicolumn{2}{c}{ Total } \\
\hline Etapa de evaluación & 1 & Recuento & 30 & 20 & 50 \\
\cline { 2 - 6 } & & \% dentro de Verificación & $60.0 \%$ & $40.0 \%$ & \\
\cline { 2 - 6 } & 2 & Recuento & 14 & 36 & 50 \\
\cline { 2 - 6 } & & \% dentro de Verificación & $28.0 \%$ & $72.0 \%$ & \\
\hline Total & Recuento & 44 & 56 & 100 \\
\hline
\end{tabular}

Fuente: Proaño (2022) 


\section{Figura 7}

Contraste del criterio gestión sostenible antes y después de la intervención

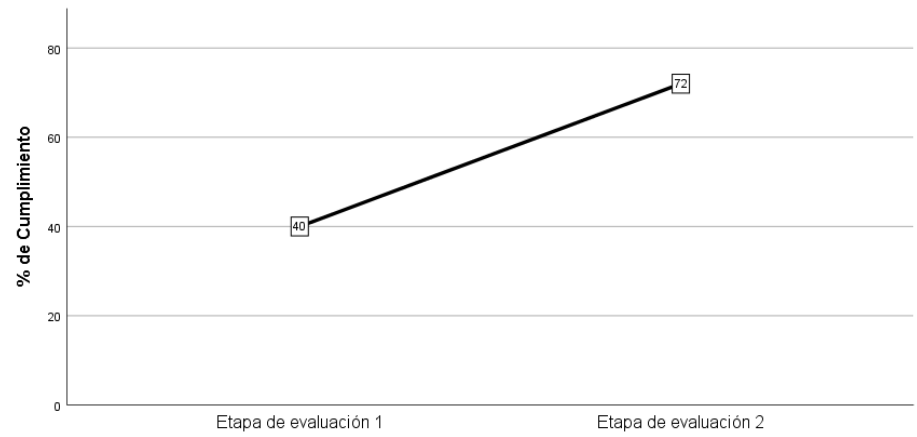

Fuente: Proaño (2022)

La gestión sostenible requiere de la implementación de 50 acciones verificables, sin embargo, se cumplen con 20 (40\%) en la evaluación 1. Este criterio se relaciona con los objetivos nueve, once, doce, trece, dieciséis y diecisiete de desarrollo sostenible (Global Sustainable Tourism Council, 2019). Lo que permite identificar que se requiere tomar conciencia de las amenazas y los riesgos que implican las actividades turísticas Martínez \& Blanco (2013); sin embargo, luego de la intervención (evaluación 2) se aprecia el cumplimiento de 36 acciones ( $72 \%$ ), existiendo un incremento del $32 \%$ lo que evidencia la relevancia de la investigación acción y facilita apreciar que el turismo sostenible no es una tipología sino un modelo de gestión.

Tabla 2

Tabla cruzada de verificación del criterio sostenibilidad socioeconómica

\begin{tabular}{lll|r|r|r} 
& & No & Si & \multicolumn{2}{c}{ Total } \\
\hline Etapa de evaluación & 1 & Recuento & 18 & 14 & 32 \\
\cline { 2 - 6 } & & \% dentro de Verificación & $56.3 \%$ & $43.8 \%$ & \\
\cline { 2 - 6 } & 2 & Recuento & 12 & 20 & 32 \\
\cline { 2 - 6 } & & $\%$ dentro de Verificación & $37.5 \%$ & $62.5 \%$ & 64 \\
\hline Total & Recuento & 30 & 34 & 6 \\
\hline
\end{tabular}

Fuente: Proaño (2022)

\section{Figura 8}

Contraste del criterio sostenibilidad socioeconómica antes y después de la intervención

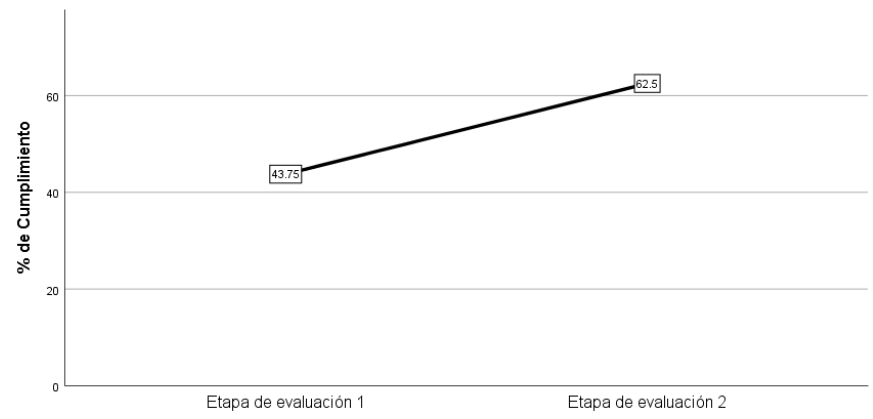

Fuente: Proaño (2022) 
El criterio impactos socio-económicos se relaciona con los objetivos uno, tres, cuatro, cinco, ocho, nueve, diez, once, doce y dieciséis de desarrollo sostenible (Global Sustainable Tourism Council, 2019) y el estudio evidencia el cumplimiento de 14 $(43,75 \%)$ de los 32 indicadores en la evaluación 1, por lo que se requería de un modelo integrado de desarrollo, donde el control directo en la planificación y gestión lo tenga la comunidad (Inostroza, 2008), lo que se evidencia en la evaluación 2 al alcanzar 62,5\%, siendo el porcentaje de mejoramiento el 18,75\%, es decir, el diagnóstico es un proceso importante para la toma de decisiones de mejoramiento de la administración y gestión.

Tabla 3

Tabla cruzada de verificación del criterio sostenibilidad cultural

\begin{tabular}{lll|r|r|r} 
& & No & Si & \multicolumn{2}{c}{ Total } \\
\hline Etapa de evaluación & 1 & Recuento & 18 & 8 & 26 \\
\cline { 2 - 6 } & & \% dentro de Verificación & $69.2 \%$ & $30.8 \%$ & \\
\cline { 2 - 6 } & 2 & Recuento & 12 & 14 & 26 \\
\cline { 2 - 6 } & & \% dentro de Verificación & $46.2 \%$ & $53.8 \%$ & 52 \\
\hline Total & Recuento & 30 & 22 & 5 \\
\hline
\end{tabular}

Fuente: Proaño (2022)

Figura 9

Contraste del criterio sostenibilidad cultural antes y después de la intervención

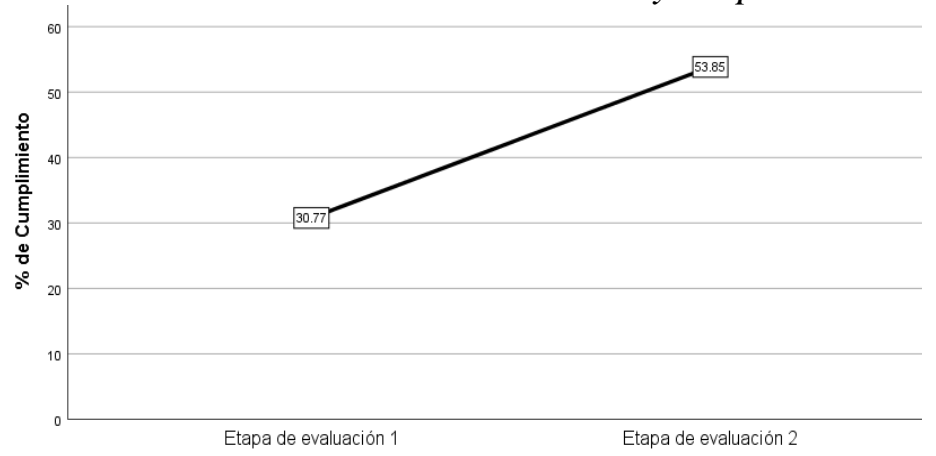

Fuente: Proaño (2022)

En el criterio impactos culturales se cumple con 8 (30,77\%) de los 26 indicadores, que se vinculan a los objetivos cuatro, once, doce y dieciséis de desarrollo sostenible (Global Sustainable Tourism Council, 2019), lo que expone que el patrimonio cultural franquea con la presión para ser adaptado a un uso turístico y se requiere un equilibrio entre ambos (Velasco, 2009), sin embargo en la evaluación 2 se apreció el incremento del 23,08\% en este criterio, debido a la creación de 64 instrumentos para la planificación, gestión y control que contribuyen a todos los criterios evaluados. 


\section{Tabla 4}

Tabla cruzada de verificación del criterio sostenibilidad del medio ambiente

\begin{tabular}{|c|c|c|c|c|c|}
\hline & & & No & $\mathrm{Si}$ & \\
\hline \multirow[t]{4}{*}{ Etapa de evaluación } & \multirow[t]{2}{*}{1} & Recuento & 57 & 8 & 65 \\
\hline & & \% dentro de Verificación & $87.7 \%$ & $12.3 \%$ & \\
\hline & \multirow[t]{2}{*}{2} & Recuento & 47 & 18 & 65 \\
\hline & & $\%$ dentro de Verificación & $72.3 \%$ & $27.7 \%$ & \\
\hline Total & & Recuento & 104 & 26 & 130 \\
\hline
\end{tabular}

Fuente: Proaño (2022)

\section{Figura 10}

Contraste del criterio sostenibilidad del medio ambiente antes y después de la intervención

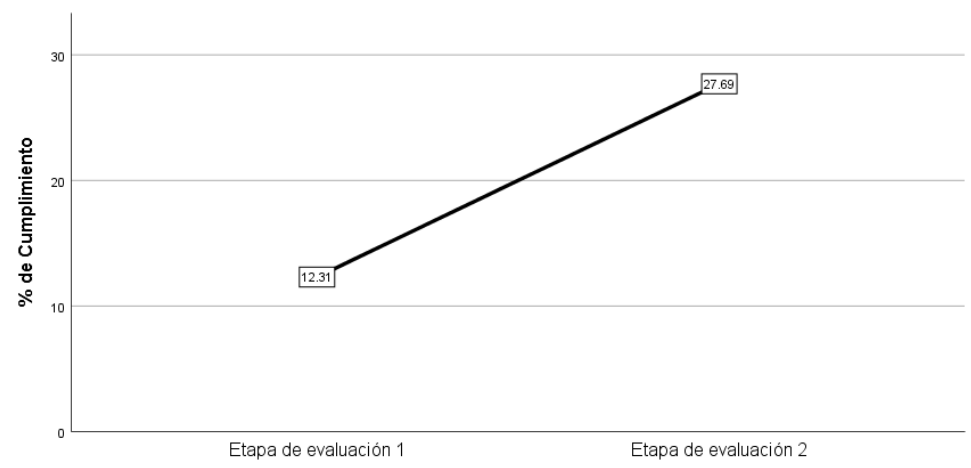

Fuente: Proaño (2022)

En el criterio impactos ambientales se cuenta con 8 (12,31\%) de los 65 indicadores durante la evaluación diagnóstica, éstos están relacionados a los objetivos tres, seis, siete, nueve, once, doce, trece, catorce y quince de desarrollo sostenible (Global Sustainable Tourism Council, 2019), siendo la gestión integrada una de las claves del desarrollo sostenible de la actividad turística (Yepes, 1999), sin embargo, en la evaluación realizada luego de la intervención se aprecia un incremento de 15,38\% en los resultados, pero queda pendiente establecer acciones urgentes para mejorar el manejo de los desechos sólidos, planes de reacción en caso de incendio y manejo de animales ferales. El diseño del modelo de gestión ha motivado al centro turístico para continuar con un proceso de mejoramiento considerando que anhelan alcanzar un reconocimiento como un distintivo sostenible en base a sus buenas prácticas.

La intervención permitió la estructuración del modelo de gestión sostenible, que se resumen en las siguientes tablas y gráficos: 


\section{Tabla 5}

Modelo de gestión resumido

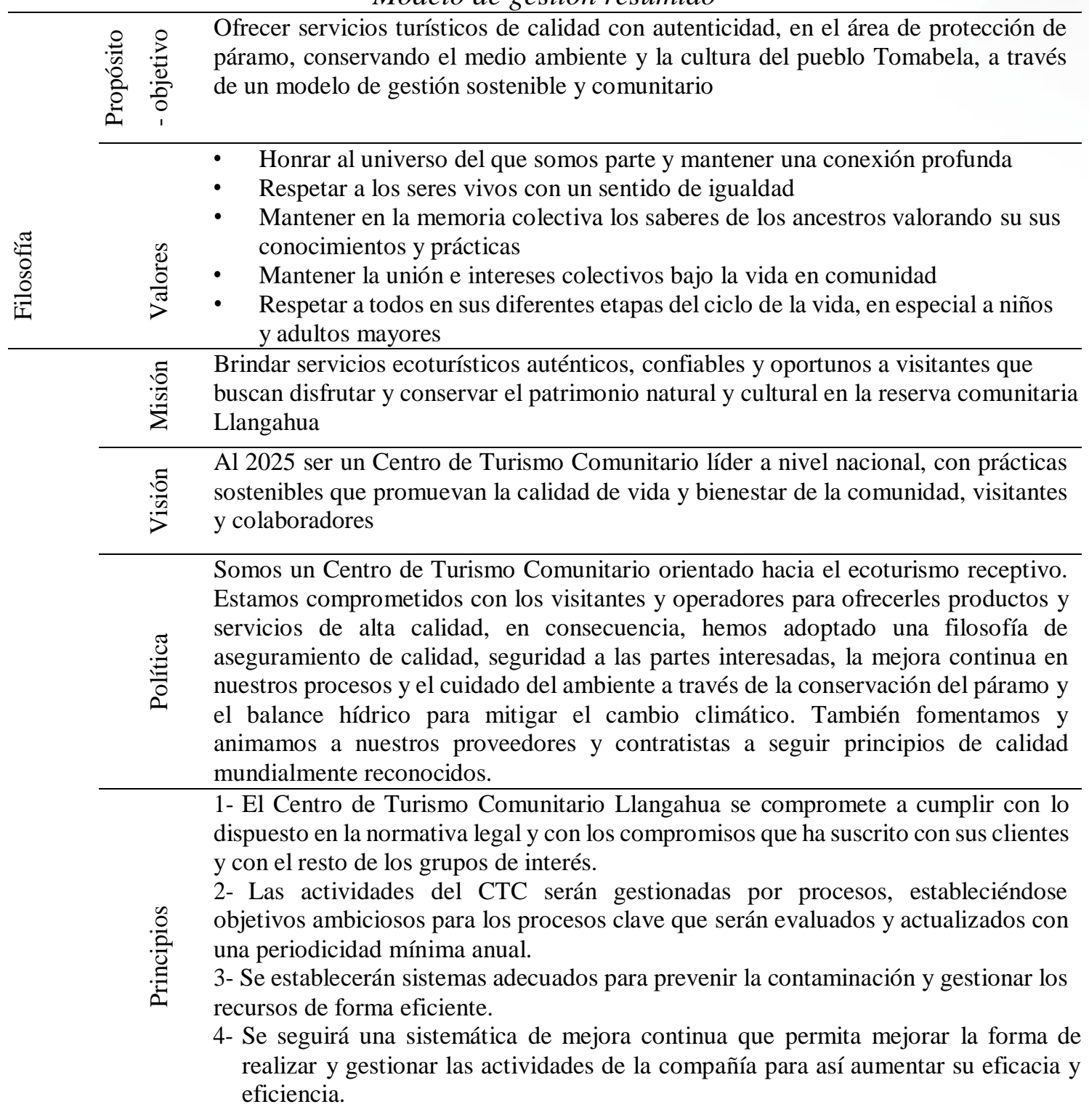

Fuente: Proaño (2022) 
Figura 11

Modelo de gestión, estrategias y programas

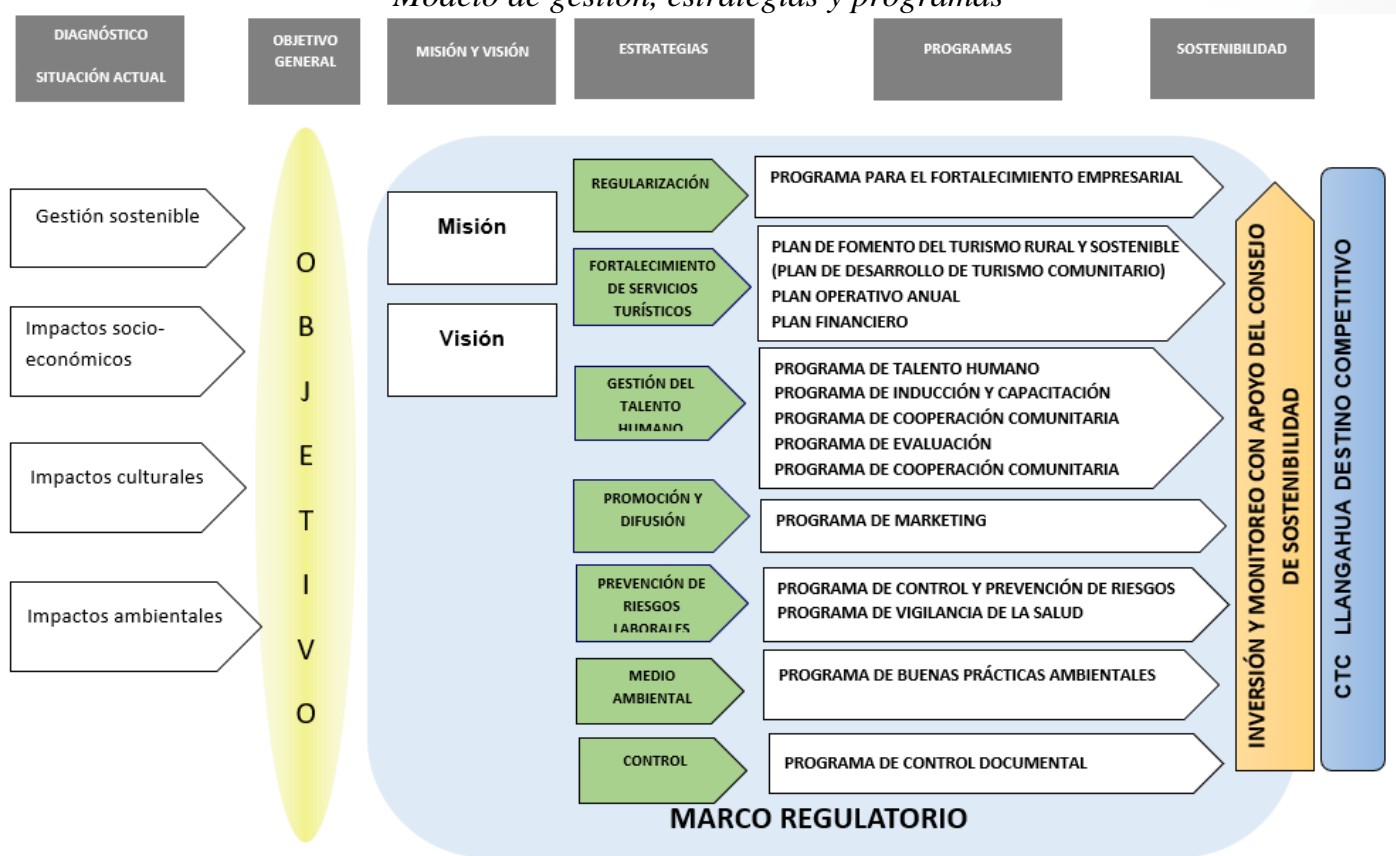

Fuente: Proaño (2021)

Tabla 6

Estrategias, programas y acciones

\begin{tabular}{|c|c|c|}
\hline ESTRATEGIAS & PROGRAMAS & ACCIONES \\
\hline \begin{tabular}{lr}
\multicolumn{3}{l}{ Regulación: Cumplir } \\
con requisitos \\
permisos para el \\
correcto y legal \\
funcionamiento \\
del Centro de \\
Turismo \\
Comunitario \\
Llangahua \\
\end{tabular} & $\begin{array}{l}\text { Programa para } \\
\text { el } \\
\text { fortalecimiento } \\
\text { empresarial }\end{array}$ & $\begin{array}{ll}\text { Instructivo } & \begin{array}{l}\text { para registros y para permisos } \\
\text { de funcionamiento del centro de turismo }\end{array} \\
\text { comunitario } & \end{array}$ \\
\hline \multirow[t]{2}{*}{$\begin{array}{lr}\text { Fortalecimiento } & \text { de } \\
\text { servicios } & \text { turísticos: } \\
\text { Fortalecer } & \text { los } \\
\text { servicios } & \text { turísticos } \\
\text { del CTC } & \end{array}$} & $\begin{array}{l}\text { Plan de fomento del } \\
\text { turismo rural y } \\
\text { sostenible (Plan de } \\
\text { desarrollo de turismo } \\
\text { comunitario) }\end{array}$ & $\begin{array}{l}\text { Diagnóstico, objetivo - propósito del CTC, valores, } \\
\text { misión, visión, política, principios. Estructura } \\
\text { organizacional del centro de turismo comunitario. } \\
\text { Estructura organizacional y responsabilidades del } \\
\text { comité o consejo de sostenibilidad. Ruta estratégica. } \\
\text { Manejo de visitantes y uso de áreas Normativa interna } \\
\text { del CTC Llangahua. }\end{array}$ \\
\hline & Plan operativo anual & $\begin{array}{l}\text { Registro, jerarquización de atractivos del MINTUR e } \\
\text { identificación requerimientos para mejorar. Plan } \\
\text { elaborado con participación comunitaria, con objetivos } \\
\text { medibles e indicadores de gestión. Informe anual con } \\
\text { medición en base a indicadores, con resultados } \\
\text { registrados y socializados. Informe de socialización } \\
\text { (acta con registro de asistencia y fotografías que } \\
\text { evidencien la socialización en la comunidad) }\end{array}$ \\
\hline
\end{tabular}


Tabla 6

Estrategias, programas y acciones (continuación)

\begin{tabular}{lr}
\hline \multicolumn{2}{c}{ ESTRATEGIAS } \\
\hline Fortalecimiento de \\
servicios turísticos: \\
Fortalecer r los \\
servicios turísticos \\
del CTC
\end{tabular}

\section{PROGRAMAS}

Plan financiero

del CTC servicios turísticos:

Plan en base al POA. Evidencia escrita de monitoreo y reporte de acciones y resultados Inventario actualizado Nombramiento de persona responsable y y/o un equipo responsable a quién se le ha asignado recursos (actas entrega - recepción). Normativa interna específica sobre alquiler de inmuebles (centro de capacitacióninterpretación). Normativa interna específica sobre bienes inmuebles y muebles. Informe anual sobre la contribución económica directa e indirecta del turismo en el destino (volumen, gasto, empleo, inversión y distribución del beneficio económico en el destino). Socialización del informe económico. Informe (documento) de la socialización del informe financiero (con acta y registro de asistentes y evidencia fotográfica)

Gestión del talento
humano:
Generar motivación
para aumentar la
Productividad
$\begin{aligned} & \text { competitividad y } \\ & \text { dentro de un } \\ & \text { adecuado } \\ & \text { laboral }\end{aligned}$

Programa de Talento Humano Manual de Talento Humano. Registros de personal permanente y contratado, indicando experiencia relevante

Procedimientos - Instructivos para

servicios. Perfiles de puesto.

Análisis de riesgos e impactos relacionados con los derechos humanos, incluida la trata de personas, la esclavitud moderna y el trabajo infantil, realizado regularmente

Programa de
limpieza y
desinfección

Instructivo para limpieza de servicios

higiénicos Instructivo para limpieza de cocina.

Instructivo de limpieza del salón comedor

Instructivo para limpieza de cabañas

Instructivo para limpieza de auditorio para reuniones (centro de interpretación)

Instructivo para mise en place

Nota: los instructivos incluyen anexos como registros, matrices, listas de verificación, etc.

\begin{tabular}{ll}
\hline $\begin{array}{l}\text { Programa } \\
\text { de }\end{array}$ & $\begin{array}{l}\text { Plan de capacitación anual } \\
\text { Registro para inducción y capacitaciones } \\
\text { inducción y } \\
\text { capacitación }\end{array}$ \\
$\begin{array}{ll}\text { Capacitación acerca del Código de Conducta para la } \\
\text { Protección de los Niños contra la Explotación Sexual en } \\
\text { Viajes y Turismo }\end{array}$ \\
\hline Programa de & Vínculos y compromiso con otros organismos del \\
cooperación & sistema turístico, productivo, ambiental o social \\
comunitaria; y, & Apoyo a sostenibilidad a empresas (capacitaciones \\
programa de & conjuntas con empresas de la comunidad) \\
información, & Inclusión de personas de la comunidad en \\
educación y & capacitaciones con temas de Turismo (evidenciar con \\
capacitación en & registro de asistencia). Productos y artesanías locales \\
turismo para & identificados, promocionados y disponibles para la \\
residentes & venta a los visitantes en el destino
\end{tabular}


Tabla 6

Estrategias, programas y acciones (continuación)

\begin{tabular}{|c|c|}
\hline ESTRATEGIAS & PROGR \\
\hline $\begin{array}{l}\text { Gestión del talento } \\
\text { humano: } \\
\text { Generar motivación } \\
\text { para aumentar la }\end{array}$ & $\begin{array}{l}\text { Programa } \\
\text { de } \\
\text { evaluación }\end{array}$ \\
\hline 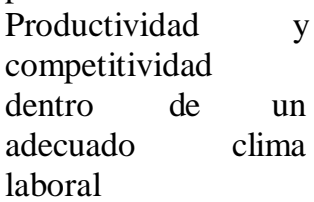 & \\
\hline
\end{tabular}

\section{ACCIONES}

Información sobre el número mensual visitantes, las variaciones estacionales, total anual y la distribución equitativa. Evaluación de proveedores. Listas de bienes y manifestaciones culturales, incluida la evaluación e indicación de vulnerabilidad. Lista de sitios y activos del patrimonio natural, indicando tipo, estado de conservación y vulnerabilidad Encuestas para medir nivel de satisfacción del cliente interno. Encuesta para medir el nivel de satisfacción del cliente externo. Evaluación de desempeño del personal. Bitácora de quejas sugerencias y felicitaciones Gestión de quejas, reclamos y sugerencias Informes de resultados y de logro de indicadores

Evaluación de impacto, que abarcan los impactos ambientales, económicos y socioculturales

\begin{tabular}{|c|c|}
\hline $\begin{array}{l}\text { Promoción } \\
\text { difusión: Aumentar el } \\
\text { índice de ventas a } \\
\text { través de la } \\
\text { notoriedad y } \\
\text { posicionamiento del } \\
\text { CTC en el mercado } \\
\text { turístico }\end{array}$ & $\begin{array}{l}\text { Programa } \\
\text { de } \\
\text { marketing }\end{array}$ \\
\hline
\end{tabular}
Proyecto de marketing del CTC. Selección de los mercados objetivos tomando en cuenta los patrones de visita, el impacto de las actividades y las necesidades del destino

Manual de uso de la marca. Instructivo para difusión, publicidad y promoción Instructivo para manejo de redes sociales, seguimiento y generación de información. Material interpretativo informativo sobre sitio $\mathrm{y}$ en formatos accesibles en idiomas relevantes y que identifica la importancia y sensibilidad / fragilidad de los sitios

\begin{tabular}{|c|c|c|}
\hline \multirow[t]{2}{*}{$\begin{array}{l}\text { Prevención de riesgos } \\
\text { laborales: Identificar } \\
\text { las actividades } \\
\text { necesarias para aislar, } \\
\text { mitigar o paliar los } \\
\text { riesgos laborales y } \\
\text { ambientales } \\
\text { relacionados con los } \\
\text { puestos de trabajo }\end{array}$} & $\begin{array}{l}\text { Programa de } \\
\text { control y } \\
\text { prevención de } \\
\text { riesgos }\end{array}$ & $\begin{array}{l}\text { Identificar las actividades necesarias para aislar, mitigar } \\
\text { o paliar los riesgos laborales y ambientales relacionados } \\
\text { con los puestos de trabajo de la empresa. Reducción de } \\
\text { riesgos, gestión de crisis y respuesta a emergencias para } \\
\text { el turismo en el destino. Plan de contingencia en caso de } \\
\text { desastres naturales, terrorismo y el agotamiento de los } \\
\text { recursos Procedimientos de comunicación identificados } \\
\text { para su uso durante y después de una emergencia. } \\
\text { Programa de entrega local de información y formación } \\
\text { en gestión de riesgos y crisis }\end{array}$ \\
\hline & $\begin{array}{l}\text { Programa de } \\
\text { vigilancia de la } \\
\text { salud }\end{array}$ & $\begin{array}{l}\text { Cumplimiento de normativa en base a programación con } \\
\text { plazos y responsables. Servicios de seguridad y salud } \\
\text { están bien establecidos y activos en el destino }\end{array}$ \\
\hline
\end{tabular}


Tabla 6

Estrategias, programas y acciones (continuación)

\begin{tabular}{|c|c|c|}
\hline ESTRATEGIAS & PROGRAMAS & ACCIONES \\
\hline $\begin{array}{l}\text { Medio ambiente: } \\
\text { Prevenir y reducir los } \\
\text { impactos ambientales } \\
\text { negativos de la } \\
\text { actividad turística a } \\
\text { través de mejoras } \\
\text { progresivas en los } \\
\text { procesos }\end{array}$ & $\begin{array}{l}\text { Programa de buenas } \\
\text { prácticas } \\
\text { ambientales }\end{array}$ & $\begin{array}{l}\text { Código de práctica para operadores y guías turísticos } \\
\text { Cooperación con organismos locales de conservación } \\
\text { para identificar riesgos ambientales asociados con el } \\
\text { turismo y medidas para reducirlos. Programa de } \\
\text { monitoreo de la calidad del agua Mejoras progresivas en } \\
\text { los procesos. Proyecto para erradicar y controlar } \\
\text { especies invasoras Proyecto para mejoramiento de } \\
\text { manejo de desechos sólidos y aguas residuales. Gestión } \\
\text { de desperdicios de alimentos Sistema de reciclaje. } \\
\text { Mecanismos para utilizar los ingresos del turismo para } \\
\text { apoyar la conservación de los activos naturales } \\
\text { Inversión en energía renovable y porcentaje de provisión/ } \\
\text { consumo total }\end{array}$ \\
\hline
\end{tabular}

Contenedores adecuados para la eliminación separada de residuos. Provisión de orientación y apoyo para el monitoreo y la reducción del uso del agua. Información promovida a los visitantes sobre opciones alternativas de transporte hacia y dentro del destino (bicicletas). Datos sobre el uso de medios de transporte alternativos por visitantes. Mejora y promoción de ciclismo y oportunidades para caminar / hacer caminatas

$\begin{array}{ll}\text { Control: Mantener un } & \text { Programa de } \\ \text { registro y control } & \text { control documental } \\ \text { documental } & \end{array}$
c omo evidencia de la gestión, fuente de consulta, apoyo e historia del proceso de mejoramiento continuo

Fuente: Proaño (2021)
Manejo documental y archivo: Lista de documentos con códigos asignados. Documentos archivados. Matrices de documentos: actas para reunión, anexos, guion turístico, etc.

\section{Conclusiones}

- En el centro de turismo Llangahua, la investigación acción realizada ha permitido estructurar un modelo de gestión sostenible en base a la evaluación de la administración, considerando el manejo comunitario, aunque el cumplimiento del marco regulatorio como centro de turismo comunitario está en proceso.

- El modelo de gestión desarrollado se constituye como un conjunto de estrategias para las buenas prácticas turísticas que trazan la ruta para las tácticas tendientes a alcanzar la filosofía de Llangahua, siendo este un proceso por lo que se ha realizado una segunda fase de intervención para continuar progresivamente fortaleciendo al turismo en la zona. 
- El modelo de gestión basado en la evaluación de la administración fortalece al centro turístico porque el personal y directivos comunitarios han estado involucrados en el proceso de evaluación 1 y 2 , y se les ha socializado los sesenta y cuatro instrumentos para la gestión que incluyen las herramientas para el seguimiento y control.

\section{Agradecimiento}

Agradecemos la participación de los estudiantes de sexto semestre de la carrera de Turismo, de la Universidad Técnica de Ambato, quienes hicieron posible el diseño de instrumentos del modelo de gestión en el periodo académico abril - agosto 2021 a través de Prácticas de Servicio Comunitario.

\section{Referencias bibliográficas}

Alonso, V. (marzo de 2010). Factores críticos de éxito y evaluación de la competitividad de destinos turísticos. Estudios y Perspectivas en Turismo, 19(2), 201-220. Recuperado el 17 de diciembre de 2021, de https://www.redalyc.org/pdf/1807/180713901003.pdf

Beltrán, A., Ocampo, C. \& Castillo, O. (diciembre de 2018). Sostenibilidad turística en Cartagena de indias. Una mirada a la gestión del turismo sostenible como motor impulsor del desarrollo y la inclusión social en este destino turístico. INNOVAITFIP, 3(1), 36 - 43. Recuperado el 13 de diciembre de 2021, de https://dialnet.unirioja.es/servlet/articulo?codigo $=7964680$

Cardoso, C. (mayo de 2006). Turismo Sostenible: una revisión conceptual aplicada. El Periplo Sustentable (11), 5-21. Recuperado el 10 de diciembre de 2021, de https://www.redalyc.org/pdf/1934/193420679001.pdf

Castellanos, M. E. (abril de 2021). Centro de Turismo Comunitario Llangahua. (Y. Proaño, Entrevistador)

Cayatopa Ylatoma, C. (2018). Modelo de gestión turística municipal integrada y participativa para el fortalecimiento de la oferta turística cultural. UCV-HACER. Revista de Investigación y Cultura, 7(3), 77-87. Doi: https://doi.org/10.18050/RevUCVHACER.v7n3a8

Cordero, J., Ortiz, F. \& Fonseca, Y. (enero - marzo de 2012). La auditoría de valores organizacionales, una herramienta para el desarrollo del turismo sostenible. Avances, 14(1), 73 - 83. Recuperado el 12 de diciembre de 2021, de http://www.ciget.pinar.cu/Revista/No.2012- 
1/articulos/auditoria_desarrollo_turismo.pdf

Costa, F. \& Castanhar, J. (2003). Avaliação de programas públicos: desafios conceituais e metodológicos. Revista De Administração Pública, 37(5), 969 a 992 . Obtenido de https://bibliotecadigital.fgv.br/ojs/index.php/rap/article/view/6509

Del Barco, L. (2010). Turismo comunitario en países en vías de desarrollo: Buenas prácticas para la planificación de un emprendimiento. Tesis, Universidad de Alicante, Instituto Universitario de Investigaciones Turísticas, Alicante. https://rua.ua.es/dspace/bitstream/10045/16411/1/Turismo\%20comunitario\%20 Del\%20Barco.pdf

Falcão de Oliveira. (2015). Políticas públicas de turismo en Brasil. Una evaluación en 65 municipios. Estudios y Perspectivas en Turismo, 24(1), 76-95. Obtenido de https://www.redalyc.org/pdf/1807/180732864005.pdf

García, N. (2017). Modelo de gestión para fomentar el desarrollo turístico de las comunidades manabitas, Ecuador. CULTUR: Revista de Cultura e Turismo, 11(2), 81-108. Obtenido de https://dialnet.unirioja.es/servlet/articulo?codigo $=6311570$

Global Sustainable Tourism Council. (19 de diciembre de 2019). Consejo Global de Turismo Sostenible (GSTC). Obtenido de Criterios GSTC para Destinos: https://www.gstcouncil.org/criterios-gstc/criterios-gstc-para-destinos/?lang=es

Guamán, J. \& Fabara, F. (2019). La ilustración como aporte al fortalecimiento de la cultura indígena de Tomabela. Carrera de Diseño gráfico publicitario. Ambato: Universidad Técnica de Ambato. Recuperado el 28 de abril de 2021, de https://repositorio.uta.edu.ec/bitstream/123456789/29116/1/Guam\%c3\%a1n\%20 Jessica.pdf

Huertas, N. (2016). Turismo rural comunitario como una propuesta metodológica de Innovación Social para comunidades en conflicto. Caso Montes de María. Tesis doctoral, Universidad de Girona, Girona. Obtenido de https://www.tdx.cat/bitstream/handle/10803/378657/tnch1de1.pdf?sequence=6\& isAllowed $=\mathrm{y}$

Inostroza, G. (2008). Aportes para un modelo de gestión sostenible del turismo comunitario en la región Andina. Gestión Turística (10), 77 - 90. Obtenido de http://revistas.uach.cl/pdf/gestur/n10/art06.pdf

Luna, B. \& Polo, A. (2009). Elaboración de un producto turístico comunitario en las comunidades Pucutahua y Llangahua del cantón Ambato, provincia de Tungurahua. Escuela de Ingeniería en Ecoturismo. Riobamba: Escuela Superior 
Técnica del Chimborazo. Recuperado el 26 de abril de 2021, de file:///C:/Users/usuario/Downloads/23T0208\%20.pdf

Martínez, V. \& Blanco, R. (junio de 2013). Hacia una gestión sostenible de las actividades turísticas en los espacios rurales y naturales. Revista Internacional de Organizaciones (10), 131-155. Obtenido de http://www.revistario.org/index.php/revista_rio/article/view/126/pdf

Moreta, M. (17 de noviembre de 2019). Tungurahua: Llangahua protege el agua. El Comercio. Recuperado el 27 de abril de 2021, de https://www.elcomercio.com/tendencias/tungurahua-llangahua-protege-aguaflora.html

Municipio de Copacabana. (2008). Manual de buenas prácticas en la actividad turística. Titicaca, Bolivia: Fundación CODESPA. Obtenido de https://www.codespa.org/app/uploads/manual-buenas-practicas-turismo-ruralcomunitario.pdf

Oficina Internacional de Trabajo. (2006). IV Encuentro Latinoamericano. Códigos de conducta y uso de marcas. Memoria, Departamento de creación de empleos y desarrollo de la empresa, Ginebra. Obtenido de https://www.ilo.org/wcmsp5/groups/public/---ed_emp/---emp_ent/--ifp_seed/documents/publication/wcms_117523.pdf

Pearce, D. (2016a). Destination management: plans and practitioners' perspectives in New Zealand. Tourism Planning \& Development, 13(1), 52-71. doi:10.1080/21568316.2015.1076511

Pearce, D. (2016b). Modelos de gestión de destinos. Síntesis y evaluación. Estudios y perspectivas en turismo, 25(1), 1-16. Recuperado el 19 de diciembre de 2021, de https://www.redalyc.org/pdf/1807/180743275001.pdf

Proaño Gómez, Y. (2021). Fomento del turismo rural mediante la estructuración de instrumentos para la gestión del turismo comunitario sostenible. Universidad Técnica de Ambato, Unidad de Vinculación, Ambato.

Proaño Gómez, Y. (2022). Fortalecimiento del turismo rural mediante la implementación de la gestión por procesos en Pilahuín Llangahua Fase II. Informe de Prácticas de Servicio Comunitario, Universidad Técnica de Ambato, Unidad de Vinculación, Ambato.

Ruiz, D. \& Masache, A. (2018). Turismo comunitario en Ecuador: Una visión desde la sostenibilidad. Comunidades, 2-41. Obtenido de https://www.academia.edu/37590779/Turismo_comunitario_en_Ecuador_una_v 
isi\%C3\%B3n_desde_la_sostenibilidad

Sarasa, J. (2014). Un proyecto de turismo rural para el desarrollo local. Xiloca: revista del Centro de Estudios del Jiloca (42), 101-122. http://www.xiloca.org/data/Bases\%20datos/Xiloca/X42_101_122.pdf

Solís, G. (2017). Caracterización y plan de manejo de residuos sólidos, en la comunidad de Llangahua, Tungurahua. Ingeniería en Biotecnología Ambiental. Riobamba: Escuela Superior Politécnica del Chimborazo. Recuperado el 27 de abril de 2021, de http://dspace.espoch.edu.ec/bitstream/123456789/7034/1/236T0281.pdf

UNESCO. (2021). Principales agendas globales de desarrollo sostenible. Loja, Ecuador: UNESCO.

Unidad de Movimientos Indígenas y Campesinos de Tungurahua. (2016). Fondo de Páramos Tungurahua y Lucha contra la Pobreza. Obtenido de Corporación de Organizaciones Populares y Campesinas Cristóbal Pajuña - COCP: https://fondotungurahua.org.ec/cocp

UNWTO. (2020). El 100\%de los destinos del mundo ha restringido ya los viajes a causa de la COVID-19, informa la OMT. Madrid, España: World Tourism Organization. https://www.unwto.org/es/news/covid-19-restricciones-viajes

Velasco González, M. (2009). Gestión turística del patrimonio cultural: Enfoques para un desarrollo sostenible del turismo cultural. Cuadernos de Turismo (23), 237-254. Obtenido de https://revistas.um.es/turismo/article/view/70121

Yepes, V. (1999). Las playas en la gestión sostenible del Litoral. Cuadernos de Turismo (4), 89-110. Obtenido de https://revistas.um.es/turismo/article/view/22881

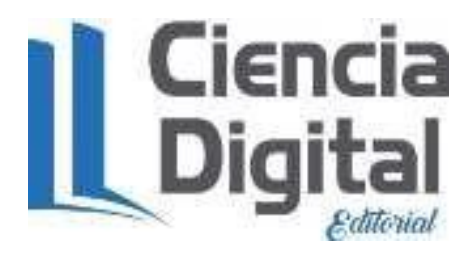


El artículo que se publica es de exclusiva responsabilidad de los autores y no necesariamente reflejan el pensamiento de la Revista Conciencia Digital.

\section{Ciencia \\ LDigital}

El artículo queda en propiedad de la revista y, por tanto, su publicación parcial y/o total en otro medio tiene que ser autorizado por el director de la Revista Conciencia Digital.

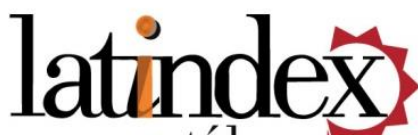

catálogo

ठ

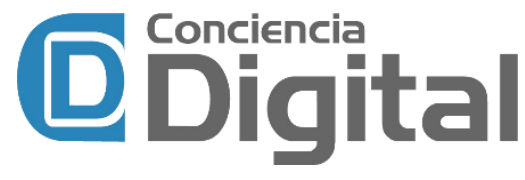

Indexaciones

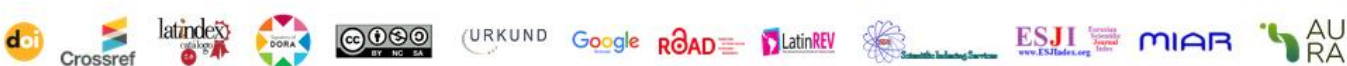

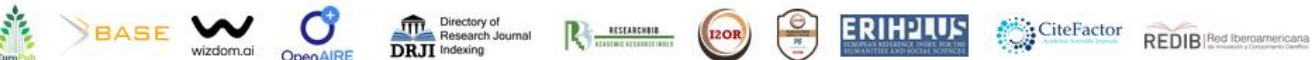

